\title{
A BIVARIATE RANDOM ENVIRONMENTAL STRESS MODEL
}

\author{
PUSHPA L. GUPTA, ${ }^{*}$ University of Maine \\ R. D. GUPTA, ${ }^{* *}$ University of New Brunswick
}

\begin{abstract}
We study the relative errors in reliability measures for the series system, under the wrong assumption of independence incorporating environmental effects, when the Marshall and Olkin model with environmental effects should be used.
\end{abstract}

\section{Introduction}

Suppose that in a two-component system, the component lifetimes under a fixed environment follow Marshall and Olkin's (1967) bivariate exponential distribution with parameters $\eta \lambda_{1}, \eta \lambda_{2}$ and $\eta \lambda_{12}$. Since the operating environment for the system is usually random, we assume that $\eta$ has a gamma distribution with parameters $a$ and $b$. Then the unconditional joint survival function of the component lifetimes $Y_{1}$ and $Y_{2}$ is

$$
b^{a}\left[b+\lambda_{1} y_{1}+\lambda_{2} y_{2}+\lambda_{12} \max \left(y_{1}, y_{2}\right)\right]^{-a} \text {. }
$$

If the two component lifetimes are independent, the model (1.1) reduces to that of Lindley and Singpurwalla (1986). While our full-length paper was presented at the 46th session of the ISI in Tokyo, Japan, 1987, it has been pointed out that certain properties of (1.1) have been established by Bandyopadhyay and Basu (1990). We therefore omit all results which duplicate theirs. Some additional properties of (1.1) are:

(i) $P\left(Y_{1}=Y_{2}\right)=\lambda_{12} /\left(\lambda_{1}+\lambda_{2}+\lambda_{12}\right)=\lambda_{12} / \lambda$, independent of the environmental effect.

(ii)

$$
\bar{F}\left(y_{1} \mid y_{2}\right)=\left\{\begin{array}{l}
{\left[\frac{b+\left(\lambda_{2}+\lambda_{12}\right) y_{2}}{b+\lambda_{1} y_{1}+\left(\lambda_{2}+\lambda_{12}\right) y_{2}}\right]^{a+1}-\left(\frac{\lambda_{12}}{\lambda_{2}+\lambda_{12}}\right)\left[\frac{b+\left(\lambda_{2}+\lambda_{12}\right) y_{2}}{b+\lambda y_{2}}\right]^{a+1}, \quad y_{1}<y_{2}} \\
\left(\frac{\lambda_{2}}{\lambda_{2}+\lambda_{12}}\right)\left(\frac{b+\left(\lambda_{2}+\lambda_{12}\right) y_{2}}{b+\left(\lambda_{1}+\lambda_{12}\right) y_{1}+\lambda_{2} y_{2}}\right)^{a+1}, \quad y_{1} \geqq y_{2} .
\end{array}\right.
$$

$$
E\left(Y_{1}^{i} Y_{2}^{j}\right)=\frac{b^{i+j} \Gamma(a-i-j)}{\Gamma(a)} E\left(X_{1}^{i} X_{2}^{j}\right), \quad a>i+j
$$

where $X_{1}, X_{2}$ follow the Marshall and Olkin (MO) model. For $E\left(X_{1}^{i} X_{2}^{j}\right)$ see Barlow and Proschan (1975).

(iv) $P\left(T_{1}>Y_{2}\right)=\lambda_{2} / \lambda$, independent of the environmental effect.

Received 28 November 1989; revision received 13 March 1990.

* Postal address: Department of Mathematics, University of Maine, Orono, ME 04469-0122, USA.

** Postal address: Division of Mathematics, Engineering and Computer Science, University of New Brunswick, Saint John, NB, Canada E2L 4LS.

Partially supported by NSERC Research Grant \#A-4850. 
(v) The conditional failure rate is given by

$$
\begin{aligned}
\Lambda\left(t_{1} \mid t_{2}\right) & =\frac{\text { conditional density of } Y_{1} \text { at } t_{1} \text { given } Y_{2}=t_{2}}{\text { conditional survival function of } Y_{1} \text { at } t_{1} \text { given } Y_{2}=t_{2}} \\
& =\frac{\lambda_{1}(a+1)}{\left[b+\left(\lambda_{2}+\lambda_{12}\right) t_{2}+\lambda_{1} t_{1}\right]}\left[1-\left(\frac{\lambda_{12}}{\lambda_{2}+\lambda_{12}}\right)\left(\frac{b+\left(\lambda_{2}+\lambda_{12}\right) t_{2}+\lambda_{1} t_{1}}{b+\lambda t_{2}}\right)^{a+1}\right], \quad t_{1}<t_{2} \\
& =\frac{\left(\lambda_{1}+\lambda_{12}\right)(a+1)}{\left(b+\left(\lambda_{1}+\lambda_{12}\right) t_{1}+\lambda_{2} t_{2}\right)}, \quad t_{1}>t_{2} .
\end{aligned}
$$

\section{Reliability measures and their relative errors}

When components are installed in a series system, a common assumption is that their lifetimes are independent and exponentially distributed. The erroneous assumption of independence when the lifetimes follow a multivariate distribution may lead to invalid inferences.

The statistical analysis of a system should be based on field data in the operating environment rather than laboratory data in a fixed environment. Even in the latter case, an error in the analysis for the series system can arise because of the non-identifiability problem described in Tsiatis (1975). The relative error in the reliability function is defined as: $\left[\bar{F}_{\mathrm{MO}}(t)-\bar{F}_{\mathrm{LS}}(t)\right] / \bar{F}_{\mathrm{LS}}(t)$, where MO stands for the Marshall and Olkin model and LS stands for the Lindley and Singpurwalla model. The relative errors in the failure rate and the mean residual life can be similarly defined.

Although the three reliability measures, namely the reliability function, the failure rate and the mean-residual life are equivalent, their relative errors do not exhibit such a property. However, in our case it turns out that (relative error in failure rate +1 ) (relative error in mean residual life +1$)=1$ (see Table $1(b)$ ). Tables $1(a)$ and $1(b)$ list the reliability measures and the relative errors.

TABLE 1(a)

Reliability measures for the two-component series system

\begin{tabular}{lccc}
\hline \multicolumn{1}{c}{ model } & $\begin{array}{c}\text { reliability } \\
\text { function }\end{array}$ & $\begin{array}{c}\text { failure } \\
\text { rate }\end{array}$ & $\begin{array}{c}\text { mean-residual } \\
\text { life }\end{array}$ \\
\hline Lindley and Singpurwalla & {$\left[\frac{b}{b+\left(\lambda_{1}+\lambda_{2}\right) t}\right]^{a}$} & $\frac{a\left(\lambda_{1}+\lambda_{2}\right)}{b+\left(\lambda_{1}+\lambda_{2}\right) t}$ & $\frac{b+\left(\lambda_{1}+\lambda_{2}\right) t}{(a-1)\left(\lambda_{1}+\lambda_{2}\right)}, a>1$ \\
$\begin{array}{l}\text { Marshal and Olkin } \\
\text { (with environmental } \\
\text { effect) }\end{array}$ & {$\left[\frac{b}{b+\lambda t}\right]^{a}$} & $\frac{a \lambda}{b+\lambda t}$ & $\frac{b+\lambda t}{(a-1) \lambda}, a>1$ \\
\hline
\end{tabular}

TABLE 1(b)

Relative error in reliability measures under the assumption of Lindley and Singpurwalla

\begin{tabular}{lccc}
\hline \multicolumn{1}{c}{ true model } & $\begin{array}{c}\text { reliability } \\
\text { function }\end{array}$ & $\begin{array}{c}\text { failure } \\
\text { rate }\end{array}$ & $\begin{array}{c}\text { mean-residual } \\
\text { life }\end{array}$ \\
\hline $\begin{array}{l}\text { Marshall and } \\
\text { Olkin (with } \\
\text { environmental } \\
\text { effect) }\end{array}$ & {$\left[\frac{b+\left(\lambda_{1}+\lambda_{2}\right) t}{b+\lambda t}\right]^{a}-1$} & {$\left[\frac{\lambda}{\lambda_{1}+\lambda_{2}}\right]\left[\frac{b+\left(\lambda_{1}+\lambda_{2}\right) t}{b+\lambda t}\right]-1$} & {$\left[\frac{\lambda_{1}+\lambda_{2}}{\lambda}\right]\left[\frac{b+\lambda t}{b+\left(\lambda_{1}+\lambda_{2}\right) t}\right]-1$} \\
\hline
\end{tabular}




\section{Analysis of the errors}

1. The relative error in reliability is negative and decreases from 0 to $\left\{\left(\left(\lambda_{1}+\lambda_{2}\right) / \lambda\right)^{a}-1\right\}$ as a function of $t\left(\lambda_{12}\right.$ fixed) or $\lambda_{12}(t$ fixed). Thus in this case the assumption of independence leads to overassessing the reliability.

2. The relative error in failure rate is positive, decreasing from $\lambda_{12} /\left(\lambda_{1}+\lambda_{2}\right)$ to 0 as a function of $t\left(\lambda_{12}\right.$ fixed) and increasing as a function of $\lambda_{12}(t$ fixed). The assumption of independence leads to underassessment of the failure rate.

3. The relative error in the mean-residual life is negative, decreasing as a function of $\lambda_{12}(t$ fixed) and increasing as a function of $t$ ( $\lambda_{12}$ fixed) from $-\lambda_{12} / \lambda$ to 0 . The wrong assumption in this case leads to overassessment of the mean-residual life.

4. The relative errors in mean-residual life and failure rate are independent of the parameter $a$, while the relative error in reliability is a function of both $a$ and $b$. This is due to the fact that the expressions. for the mean-residual life for the two models are such that the effect of $a$ cancels out. The case for the two failure rates is similar. Although the three reliability measures can be obtained from each other, the three relative errors in general do not exhibit such a property.

5. The effect of the environment on the Marshall and Olkin model can be shown as follows:

\begin{tabular}{c|c|c} 
& $b<a(E(\eta)>1)$ & $b>a(E(\eta)<1)$ \\
\hline$t<(a-b) / \lambda$ & $\begin{array}{c}\text { Harsher } \\
\text { (decreased reliability) }\end{array}$ & $\begin{array}{c}\text { Gentler } \\
\text { (increased reliability) }\end{array}$ \\
\hline$t>(a-b) / \lambda$ & $\begin{array}{c}\text { Gentler } \\
\text { (increased reliability) }\end{array}$ & $\begin{array}{c}\text { Gentler } \\
\text { (increased reliability) }\end{array}$
\end{tabular}

Normally a harsher (gentler) environment would suggest that $\eta>1(\eta<1)$, as noted by Lindley and Singpurwalla. In the case of $E(\eta)>1$, harsher and gentler states will depend on the age of the system.

\section{References}

BANDYopadhyay, D. AND BASU, A. P. (1990) On generalization of a model by Lindley and Singpurwalla. Adv. Appl. Prob. 22, 498-500.

Barlow, R. E. AND Proschan, F. (1975) Statistical Theory of Reliability and Life Testing: Probability Models, Holt, Rinehard and Winston, New York.

GuptA, P. L. AND Gupta, R. D. (1987) Relative error in systems reliability. Proc. 47th Session Internat. Statist. Inst., Tokyo, Japan.

Lindley, D. V. ANd Singpurwalla, N. D. (1986) Multivariate distributions for the life lengths of components of a system sharing a common environment. J. Appl. Prob. 23, 418-431.

Marshall, A. W. AND Olkin, I. (1967) A multivariate exponential distribution. J. Amer. Statist. Assoc. 62, 30-40.

Tsiatis, A. (1975) A non-identifiability aspect of the problem of competing risks. Proc. Nat. Acad. Sci. 72, 20-22. 